\title{
A full vectorial mapping of nanophotonic light fields
}

\author{
B. le Feber ${ }^{1,2}$, J. E. Sipe ${ }^{3}$, M. Wulf ${ }^{2,4}$, L. Kuipers ${ }^{2,5}$ and N. Rotenberg ${ }^{2,6}$
}

\begin{abstract}
Light is a union of electric and magnetic fields, and nowhere is the complex relationship between these fields more evident than in the near fields of nanophotonic structures. There, complicated electric and magnetic fields varying over subwavelength scales are generally present, which results in photonic phenomena such as extraordinary optical momentum, superchiral fields, and a complex spatial evolution of optical singularities. An understanding of such phenomena requires nanoscale measurements of the complete optical field vector. Although the sensitivity of nearfield scanning optical microscopy to the complete electromagnetic field was recently demonstrated, a separation of different components required a priori knowledge of the sample. Here, we introduce a robust algorithm that can disentangle all six electric and magnetic field components from a single near-field measurement without any numerical modeling of the structure. As examples, we unravel the fields of two prototypical nanophotonic structures: a photonic crystal waveguide and a plasmonic nanowire. These results pave the way for new studies of complex photonic phenomena at the nanoscale and for the design of structures that optimize their optical behavior.
\end{abstract}

The advent of metamaterials and structures with a large response to the optical magnetic field ushered in a new age of near-field microscopy, where the ability to measure only electric near fields is no longer sufficient. Many nanoscopic structures, such as split ring resonators ${ }^{1,2}$, dielectric Mie scatterers ${ }^{3-6}$, and even simple plasmonic holes $^{7,8}$, have an optical response that depends on the full electromagnetic field. Likewise, measurements of many nanoscale photonic phenomena, such as superchiral fields ${ }^{9,10}$ or extraordinary spin and orbital angular momentum ${ }^{11-13}$, require access to both the electric $\mathbf{E}$ and magnetic $\mathbf{H}$ fields.

Motivated by this demand, there have been a number of efforts to extend the capability of near-field scanning optical microscopes (NSOMs) beyond the traditional measurements of $\mathbf{E}^{14}$. Proof-of-concept measurements of $\mathbf{H}$ at the nanoscale have relied on specially designed nearfield probes ${ }^{15,16}$; however, these are difficult to fabricate

\footnotetext{
Correspondence: B. Feber (boris.le.feber@prodrive-technologies.com)

${ }^{1}$ Optical Materials Engineering Laboratory, ETH Zürich, 8092 Zurich, Switzerland ${ }^{2}$ Center for Nanophotonics, AMOLF, Science Park 104, 1098 XG Amsterdam, The Netherlands

Full list of author information is available at the end of the article.
}

and tend to measure only one component of $\mathbf{H}$. Recent strategies have therefore focused on measurements with traditional aperture probes ${ }^{17,18}$, which demonstrate that even circular apertures are simultaneously sensitive to the four in-plane components $E_{x, y}$ and $H_{x, y}{ }^{19}$.

However, a crucial challenge remains. Although a polarization-resolved NSOM measurement (see Supplementary Note 1) contains information from the four inplane components, it is encoded into only two complex signals $L_{x}$ and $L_{x}$, as shown in Fig. 1. To date, unraveling these measurements to extract the individual components of the electric and magnetic fields has not been possible without the use of additional information coming from detailed simulations of the structure being measured ${ }^{20}$, on far-field optical beams ${ }^{21}$ or a symmetry plane where one component is identically zero ${ }^{22}$. At best, numerical simulations can be used to determine the spatial evolution of $|\mathbf{E}|^{2}$ and $|\mathbf{H}|^{2}$ near nanophotonic structures but not separate electromagnetic components or their phases ${ }^{23}$. Here, we show how to simultaneously extract $E_{x}, E_{y}, H_{x}$, and $H_{y}$ from a single two-channel NSOM measurement with no a priori knowledge of the nanophotonic structures being measured. By inserting these fields into 


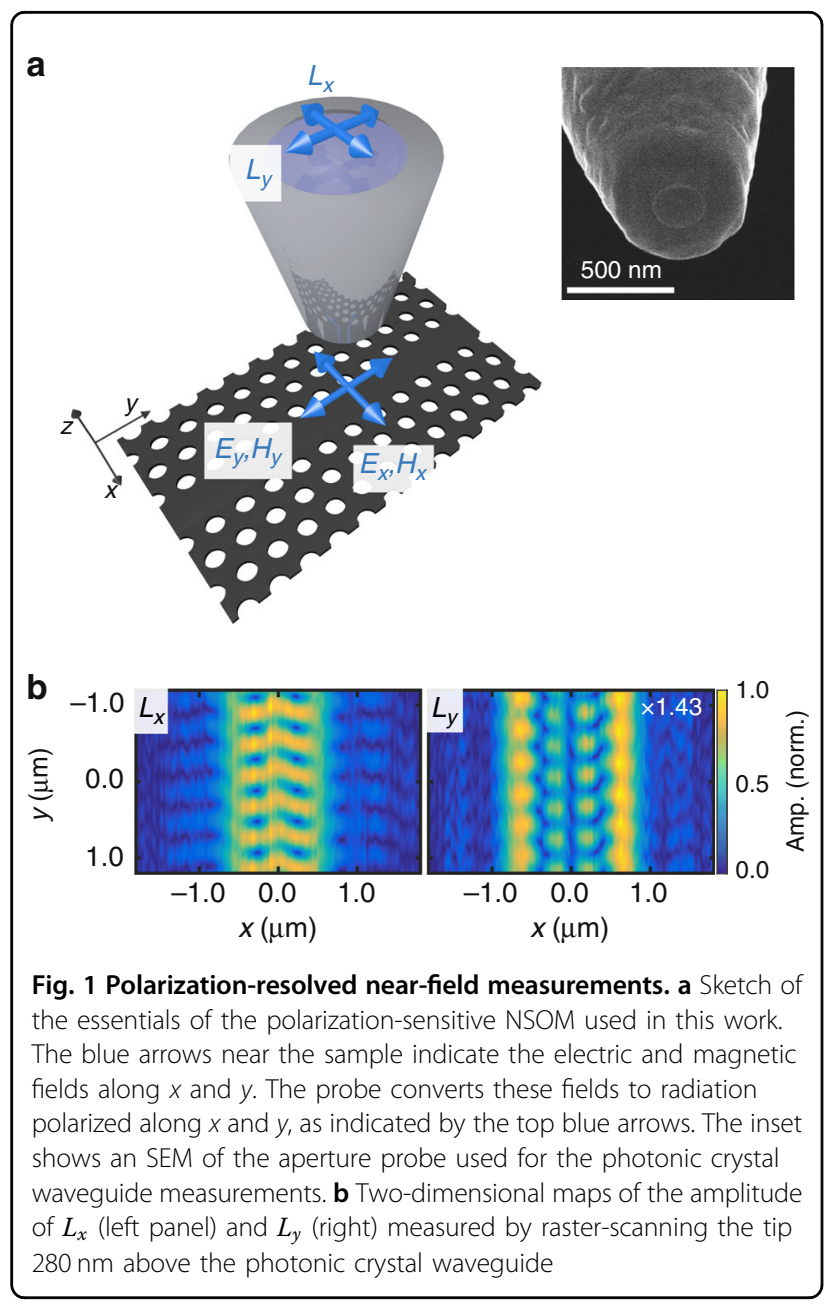

Maxwell's equations, we can obtain the two out-of-plane components $E_{z}$ and $H_{z}$ and thus achieve a full vectorial measurement of the electromagnetic near-field. The separation algorithm is robust to noise and realistic measurement conditions, as we show from exemplary NSOM experiments on both photonic crystal waveguides (PhCWs) and plasmonic nanowires.

At the heart of near-field microscopy lies the process by which the near-field probe images the light fields above a structure. For example, in the field distributions in Fig. 1b, which were measured $280 \mathrm{~nm}$ above a PhCW (Supplementary Notes 1 and 2), a representative height where the electric and magnetic field distributions contain subwavelength features and are expected to differ ${ }^{19,24}$. These images are produced as the aperture probe, which acts as an effective spatial filter, merges all four in-plane components of the sample's near-field. When this light field is highly structured with feature sizes smaller than the probe aperture, this process becomes increasingly complex, and it is less obvious exactly how efficiently and with what phase $E_{x}, E_{y}, H_{x}$, and $H_{y}$ contribute to the measured signals $L_{y}$ and $L_{x}$. In other words, calculating the transfer function of a near-field probe, which propagates the fields from the sample to a detector, has not been possible.

However, it is possible to calculate the fields that are radiated through the probe by a point dipole at position $\mathbf{r}_{0}$ of a hypothetical detector (Fig. 2a) with current density $\mathbf{j}_{\mathrm{det}} \delta\left(\mathbf{r}-\mathbf{r}_{0}\right)$. These fields, which we label $\mathbf{E}_{i}^{r}$ and $\mathbf{H}_{i}^{r}$, where $i=x, y$ indicates the orientation of $\mathbf{j}_{\mathrm{det}}$ (Fig. 2b, middle column, for the dipole in the $x$ direction), have been extensively measured and resemble those below a hole in a metal film ${ }^{14,25}$; hereafter, we take our tip to be ideally symmetric to ensure equal sensitivity to the $x$ and $y$ components of the electromagnetic field. Via the optical reciprocity theorem (ORT), we can use these probe fields to relate the sample fields $\mathbf{E}^{e}$ and $\mathbf{H}^{e}$ (Fig. 2b, left column) to dipoles at our detectors and, consequently, our measured signals (Fig. 2b, right column $)^{19,26,27}$. In other words, in this approach, $\mathbf{E}_{i}^{r}$ and $\mathbf{H}_{i}^{r}$ can be considered the spatial filters that exactly define how efficiently and with which phase different sample field components are detected. Each independent dipole orientation $x$ or $y$ is associated with all four in-plane components of the probe field, which explains why each detection channel typically contains information of all in-plane components of the sample fields. Using a specific sensing configuration ${ }^{28}$ or material composition ${ }^{16}$, it is possible to design probes that primarily detect $\mathbf{E}^{e}$ or $\mathbf{H}^{e}$ of specific near fields. However, such probes preclude complete electromagnetic measurements, so we consider aperture probes that are similarly sensitive to $\mathbf{E}^{e}$ and $\mathbf{H}^{e}$ in this work.

The image formation via the ORT can be expressed as (see Supplementary Note 4 for derivation)

$$
\begin{aligned}
L_{i}\left(\mathbf{R}_{\mathrm{tip}}\right)= & \int_{S} d S\left(\mathbf{E}^{e}(\mathbf{R}) \times \mathbf{H}_{i}^{r}\left(\mathbf{R}-\mathbf{R}_{\mathrm{tip}}\right)\right. \\
& \left.-\mathbf{E}_{i}^{r}\left(\mathbf{R}-\mathbf{R}_{\mathrm{tip}}\right) \times \mathbf{H}^{e}(\mathbf{R})\right) \cdot \hat{\mathbf{z}}
\end{aligned}
$$

where $S$ is a surface between the probe and the sample, which is $10 \mathrm{~nm}$ below the probe in this case; $\mathbf{R}_{\text {tip }}=$ $\left(x_{\text {tip }}, y_{\text {tip }}\right)$ is the position of the tip above this plane; $\mathbf{R}=$ $(x, y)$ are the coordinates of the fields on $S$; and the integral is taken over all $\mathbf{R}$. Subscript $i$ refers to the $x$ or $y$ orientation of the reciprocal dipole and not to a component of the fields. The dot product with $\hat{\mathbf{z}}$ shows that the measured image only depends on the in-plane field components. This process of image formation is shown in Fig. $2 \mathrm{~b}$, where we use the calculated probe and sample fields to predict the measured signals (see ref. ${ }^{19}$ for details on the calculations). In fact, we observe an excellent agreement between our predictions (right column, Fig. 2b) and the measurements (Fig. 1b) at $280 \mathrm{~nm}$ above 

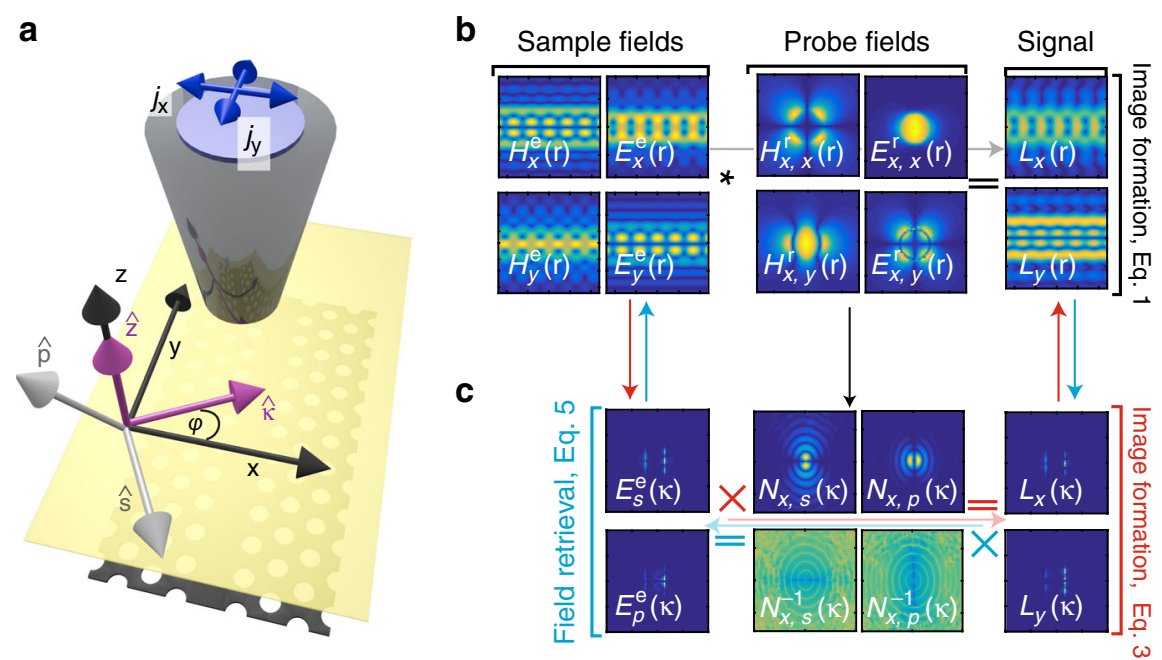

Fig. 2 Image formation and field retrieval. a Schematic of the coordinate bases and experimental setup. All fields are evaluated on a surface (transparent yellow) that completely separates the probe from the sample. The purple arrows indicate the in-plane ( $(\hat{\mathbf{k}})$ and out-of-plane $(\hat{\mathbf{z}})$ unit vectors of a plane wave on this surface, whereas the gray arrows show the corresponding unit vectors $\hat{\mathbf{s}}$ and $\hat{\mathbf{p}}$ for an upward traveling wave. $\mathbf{b}$ Real space image formation process according to Eq. 1. In real space, the measured image $L_{x, y}$ can be understood as the convolution (indicated by the asterisk sign) of the sample fields $\mathbf{E}^{e}$ and $\mathbf{H}^{e}$ and the probe fields $\mathbf{E}_{i}^{r}$ and $\mathbf{H}_{i}^{r}$, shown here for the $x$-oriented dipole $(i=x)$. c Top row: In Fourier space, the image formation process that corresponds to $(\mathbf{b})$ is described by the multiplication of the sample fields and probe response function $\mathrm{N}$, which was here calculated for an ideally symmetric probe. Bottom row: The reverse process, which results in the separated fields, therefore simply involves the multiplication of the measured signals with the inverse probe response function $\mathrm{N}^{-1}$. Note that we only show only the $x$-oriented dipole $(i=x)$ components of $\mathrm{N}$ and $\mathrm{N}^{-1}$. All maps in $(\mathbf{b}, \mathbf{c})$ show the calculated fields that are normalized to their maximum amplitude

the $\mathrm{PhCW}$, which validates this approach and the symmetry of our probes.

When we want to retrieve the sample fields, instead of studying the image formation, we face two challenges: first, we require two additional equations to match the number of unknowns; second, we must be able to invert Eq. 1 (Supplementary Note 3). To address the first challenge, we recognize that the electromagnetic field at and near the sample plane can be decomposed into a superposition of different plane waves, each of which is represented by a total wavevector $\mathbf{k}=k_{z} \hat{\mathbf{z}}+\kappa \hat{\mathbf{k}}$, where $\kappa=|\mathbf{k}|=\left|\left(k_{x}, k_{y}\right)\right|^{28}$. Here, $k_{z}$ is the out-of-plane component of the wavevector, and $k$ is the in-plane component, as shown in Fig. 2a. We can write each plane wave in the Cartesian basis $\left(E_{x}, E_{y}, E_{z}\right)$ or in terms of its $s$ - and $p$-field components $\left(E_{s+}, E_{s-}, E_{p+}, E_{p-}\right)$, which enables us to identify the upward (real $\left(k_{z}\right)>0$, subscript + ) or downward (real $\left(k_{z}\right)<0$, subscript - ) propagating waves. In principle, the full field between the sample and the probe is a combination of both upward and downward propagating fields, where the latter arise due to the interaction of the probe tip with the sample. However, this interaction is negligible if the probe and sample do not have a joint resonance ${ }^{29}$, as is indeed the case for our normal aperture probe, which has a broad spectral response ${ }^{7,28}$. Therefore, we can take the field above the sample surface to be purely upward propagating (i.e., there is no backscattering, so $E_{p-}=0$ ), which implies that we must only consider two components of the electric field and four components of the total field: $E_{s}, E_{p}, H_{s}$, and $H_{p}$, where all $s$ and $p$ components are upward propagating (i.e., $p+$ ). Finally, Maxwell's equations straightforwardly relate the electric and magnetic field components of these transverse plane waves (see Supplementary Note 5 for the derivation and conversion between the different bases)

$$
\begin{gathered}
\mathbf{E}^{e}(\mathbf{\kappa})=E_{s}^{e}(\mathbf{\kappa}) \hat{\mathbf{s}}+E_{p}^{e}(\mathbf{\kappa}) \hat{\mathbf{p}} \\
\mathbf{H}^{e}(\mathbf{\kappa})=\frac{1}{Z_{0}}\left[E_{s}^{e}(\mathbf{\kappa}) \hat{\mathbf{s}}-E_{p}^{e}(\mathbf{\kappa}) \hat{\mathbf{p}}\right]
\end{gathered}
$$

where $Z_{0}$ is the impedance of free space. Considering Eq. 1, we have reduced our problem to two unknowns $\left(E_{s}^{e}\right.$ and $E_{p}^{e}$ ) and two equations, one each for $L_{x}$ and $L_{y}$. In terms of the Fourier components, we can rewrite Eq. 1 as

$$
\frac{1}{Z_{0}}\left[\begin{array}{c}
L_{x}(\mathbf{\kappa}) \\
L_{y}(\mathbf{\kappa})
\end{array}\right]=\left[\begin{array}{ll}
N_{x, s}(\mathbf{k}) & N_{x, p}(\mathbf{k}) \\
N_{y, s}(\mathbf{k}) & N_{y, p}(\mathbf{\kappa})
\end{array}\right]\left[\begin{array}{c}
E_{s}^{e}(\mathbf{\kappa}) \\
E_{p}^{e}(\mathbf{\kappa})
\end{array}\right]
$$

where tensor $N$ is essentially the transfer matrix that maps the sample electric fields expressed in their polarization components to the detection channels associated with the $x$ - and $y$-directions. Different components of $\mathrm{N}$ are related to the Cartesian 


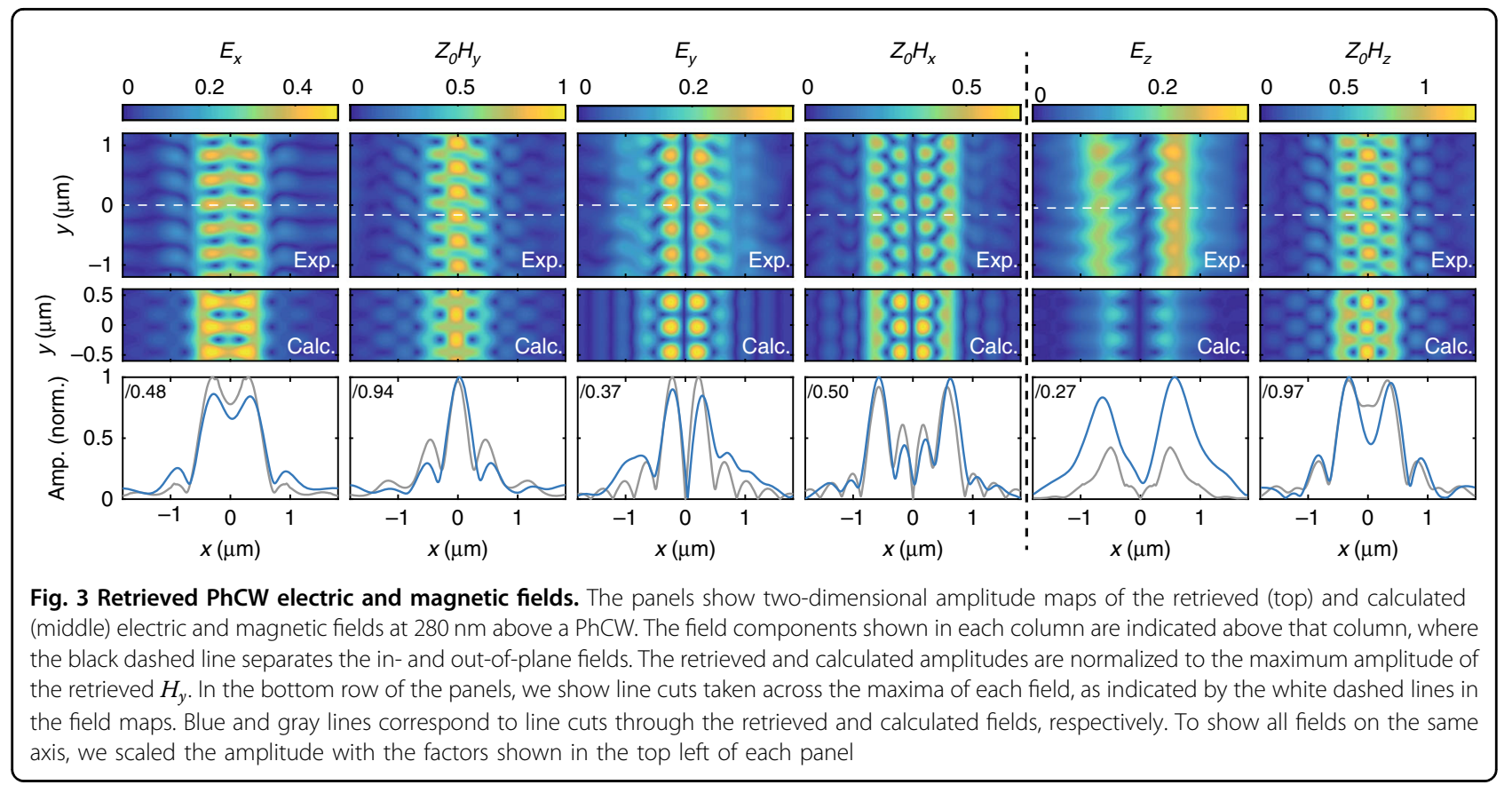

components of $\mathbf{E}_{i}^{r}$ and $\mathbf{H}_{i}^{r}$ as follows

$$
\begin{aligned}
& {\left[\begin{array}{l}
N_{i, s}(\mathbf{\kappa}) \\
N_{i, p}(\mathbf{k})
\end{array}\right]} \\
& =\left[\begin{array}{cccc}
-\frac{k_{z}}{k_{0}} \sin \phi & \frac{k_{z}}{k_{0}} \cos \phi & Z_{0} \cos \phi & Z_{0} \sin \phi \\
\cos \phi & \sin \phi & Z_{0} \frac{k_{z}}{k_{0}} \sin \phi & -Z_{0} \frac{k_{z}}{k_{0}} \cos \phi
\end{array}\right]\left[\begin{array}{c}
E_{i, x}^{r}(-\mathbf{k}) \\
E_{i, y}^{r}(-\mathbf{k}) \\
H_{i, x}^{r}(-\mathbf{k}) \\
H_{i, y}^{r}(-\mathbf{\kappa})
\end{array}\right]
\end{aligned}
$$

where $\phi$ is the angle between $\mathbf{\kappa}$ and the $x$-axis (Fig. 2a). We show the image formation process in terms of these plane wave components in the top row of Fig. 2c, which corresponds to the real space plots in Fig. 2b, where $N_{x, s}(\mathbf{\kappa})$ and $N_{x, p}(\mathbf{\kappa})$ are plotted in the middle column. These $\mathrm{N}$ maps clearly show which wavevector components contribute the most to the detected image.

Then, unraveling the near-field measurements is simply a matter of inverting $\mathrm{N}$ to obtain

$$
\left[\begin{array}{l}
E_{s}^{e}(\mathbf{\kappa}) \\
E_{p}^{e}(\mathbf{\kappa})
\end{array}\right]=\frac{1}{Z_{0}}\left[\begin{array}{ll}
N_{x, s}(\mathbf{\kappa}) & N_{x, p}(\mathbf{\kappa}) \\
N_{y, s}(\mathbf{\kappa}) & N_{y, p}(\mathbf{\kappa})
\end{array}\right]^{-1}\left[\begin{array}{c}
L_{x}(\mathbf{\kappa}) \\
L_{y}(\mathbf{\kappa})
\end{array}\right]
$$

which has a unique solution if $\operatorname{det}(\mathrm{N}) \neq 0$ for all $\mathbf{\kappa}$, as is indeed the case for our probes. Therefore, we can deconvolve a near-field measurement simply by following the steps illustrated in the bottom row of Fig. 2c. First, the measurements are Fourier transformed in the $x y$-plane to generate $L_{x, y}(\mathbf{\kappa})$, which are multiplied by $\mathrm{N}^{-1}(\mathbf{\kappa})$ to obtain $E_{s, p}^{e}(\mathbf{\kappa})$ according to Eq. 5. Then, these fields are transformed back into the Cartesian basis (Supplementary Note 5) and inverse-Fourier-transformed into the real space to arrive at the deconvolved sample fields $E_{x, y}^{e}(\mathbf{R})$ and $H_{x, y}^{e}(\mathbf{R})$. Finally, following the example of Olmon et al. $^{22}$, we use Maxwell's equations to extract the $2 \mathrm{D}$ maps of the out-of-plane electric and magnetic field components, $E_{z}^{e}(\mathbf{R})$ and $H_{z}^{e}(\mathbf{R})$, according to $E_{z}=$ $i Z_{0} k_{0}\left(\frac{\partial H_{x}}{\partial x}-\frac{\partial H_{x}}{\partial y}\right)$ and $H_{z}=-\frac{i k_{0}}{Z_{0}}\left(\frac{\partial E_{y}}{\partial x}-\frac{\partial E_{x}}{\partial y}\right)$. Because the same probe can be used for multiple measurements and $N(\mathbf{\kappa})$ is similar for probes with different aperture sizes (Supplementary Figure S13), $\mathrm{N}^{-1}(\mathbf{\kappa})$ must only be calculated once and can be used in many experiments.

The inversion of $\mathrm{N}$ (in Eq. 5) makes our deconvolution process sensitive to large-wavevector signals, although the image formation process is not (bottom and top rows of Fig. 2c, respectively). Since the experimental fields (left column, Fig. 2c) do not contain a signal at these large wavevectors, the measurement noise typically dominates there. In principle, this sensitivity to large wavevectors limits our retrieval algorithm, but it does not greatly affect its performance in practice. As we discuss below (see Fig. 4), we can simply limit the largest wavevector that we consider to the wavevector at which we still expect to find signal from the sample.

Here, we apply our algorithm to the PhCW fields shown in Fig. $1 \mathrm{~b}$ and limit ourselves to the smallest allowable wavevector range $\kappa \leq 3 k_{0}$, where $k_{0}$ is the free-space wavenumber of the light to test our retrieval procedure in the lowest resolution limit. As we discuss below and in more detail in Supplementary Note 7, the maximum allowable wavevector can by increased to $\kappa_{\max }=9 k_{0}$. The amplitudes of the separated field components are shown 
in Fig. 3 along with the theoretically calculated mode profiles. Line cuts, taken at the positions of the dashed lines are also shown, which demonstrate the excellent agreement when comparing the experimental (blue) and theoretical (gray) curves for all six electromagnetic field components. In fact, the only component for which we observe significant deviation between the predicted and measured field amplitude is $E_{z}$. We attribute this difference to the small amplitude of this component, which makes it more susceptible to errors that arise from imperfect experimental conditions, which can cause, e.g., polarization mixing. In principle, even these small errors can be improved by calculating the transfer function for the exact probe used, including minor fabrication imperfections, and not the idealized, symmetric probe here. We also observe strikingly good agreement between the calculated and retrieved phase profiles (Supplementary Figure S14). In other words, we can successfully recover the general shape of each field component and even resolve the fine features in the amplitude and phase of these in-plane fields all from a single measurement.

Our approach is not limited to dielectric structures but can be extended to nanoplasmonics. As an example, we consider a plasmonic nanowire, whose electric and magnetic near-field distributions are known to have different and nontrivial spatial dependencies ${ }^{30}$. Using our protocol, we resolve the different field components above the nanowire (see Supplementary Note 6 for details and images of the separated fields). We again observe good agreement between theoretical and

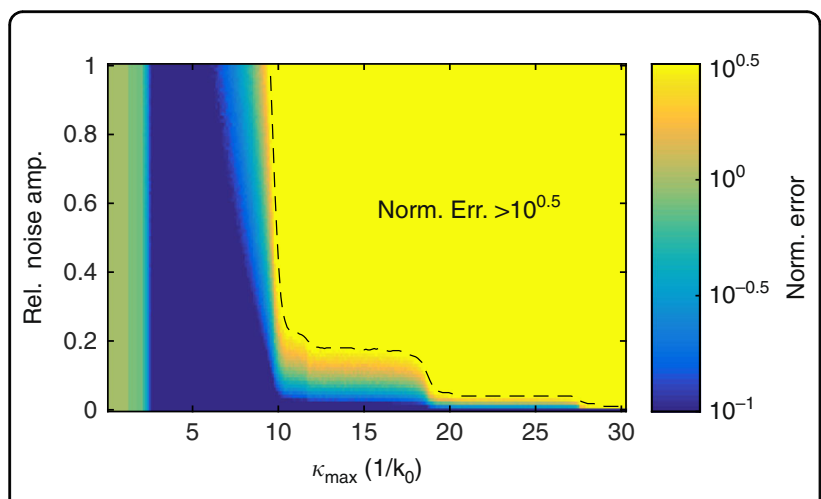

Fig. 4 Robustness of the field retrieval algorithm. Mismatch between the retrieved fields and the predicted fields (see Methods) as a function of the noise amplitude and wavevector cutoff (see text for explanation). Because small signals with a high spatial frequency can result in very large signals, which are well beyond the total intensity of the calculated fields, we saturate Fig. 4 at normalized errors larger than $10^{0.5}$ to avoid obscuring more important results at low mismatch values. Likewise, the minimum error in our calculations is at $10^{-5}$; because all values below $10^{-1}$ practically appear identical to the input fields, we saturated this plot below $10^{-1}$ measured fields, and similar to the dielectric samples, clear differences in the retrieved electric and magnetic fields from different samples are revealed (Supplementary Figure S10).

The ability of our algorithm to retrieve optical fields from measurements of a $\mathrm{PhCW}$ and a plasmonic nanowire already hints at its robustness to noise. To further explore the effect of measurement noise, we artificially add white noise to a perfect "measurement" (i.e., theoretically calculated fields with a noise level $<10^{-3}$ ) in increments until we reach a signal-to-noise ratio of unity in $L_{x, y}$. Then, we calculate the normalized error between the ideal and the retrieved optical fields (see Methods Section), which is shown in Fig. 4. More importantly, for all noise levels, we observe that the setting $\kappa_{\max }=2 k_{0}$ results in a poor field retrieval because this low limit effectively filters large portions of the input signal (Supplementary Figure S12 for the corresponding retrieved field maps and Supplementary Section S7 for additional discussion). However, up to $\kappa_{\max }=5 k_{0}$, we find nearperfect deconvolution even in cases where the noise is as large as the signal.

Finally, we note that while decreasing the probe aperture size results in a decrease in signal and a corresponding increase in resolution, it has little effect on our algorithm (Supplementary Figure S13); although higher wavevectors appear in $\mathrm{N}(\mathbf{\kappa})$ for small probe diameters, $\mathrm{N}(\boldsymbol{\kappa})$ remains nearly identical at low $\kappa$. Since the algorithm is robust even when the noise level is comparable to the signal (c.f. Figure 4), even measurements with such low-throughput probes can be deconvolved into their constituent components.

The capability to map both the electric and magnetic near-field components is important for the study and development of nanophotonic structures, particularly if the strategy is simple and robust. Our approach can be used to measure the full electric and magnetic fields near dielectric and plasmonic structures, which are increasingly necessary in a research landscape of nanoscopic structures with different electric and magnetic responses. Moreover, because the deconvolution of a full field takes only seconds when $\mathrm{N}^{-1}(\mathbf{\kappa})$ is known (Eqs. 4 and 5), our algorithm can be applied in real time. As a demonstration, we have presented the full, complex electromagnetic near-field of two nanophotonic waveguides, but we note that our approach can also be applied to other systems such as nanoantennas and cavities. For the latter case, special care must be taken with high-quality factor resonators $Q>1000$, where the interactions between the near-field probe and the photonic mode cannot be neglected and in fact can provide an independent measure of the magnetic field ${ }^{17,31}$. Measurements of nanoscale $\mathbf{E}$ and $\mathbf{H}$ have the potential to drive progress in fields such as chiral quantum 
optics $^{32,33}$, plasmonics ${ }^{34}$, and metasurfaces ${ }^{35}$, where the light-matter interactions and device performance depend on the exact form of vector near fields, often in the presence of unavoidable fabrication imperfections. A further intriguing possibility is the combination of our method with measurements of the emission of a quantum emitter placed on the probe, which map out the local density of optical states ${ }^{36,37}$ and are therefore important to quantum optical applications.

\section{Methods}

\section{Robustness to noise}

To quantify the robustness to noise of our algorithm, we compare the calculated fields to the fields retrieved from a computer-generated field map, which is obtained by applying the reciprocity theorem to the calculated fields. To this calculated mapping (such as that in Fig. 2b), we add a controlled amount of white noise. The mean amplitude of that noise relative to the maximum amplitude of the signal is shown on the $y$-axis of Fig. 4 . Next, we apply our algorithm to these noisy calculated mappings and compare the retrieved fields to the calculated fields to obtain the normalized error $\Delta=\left.\sum_{E_{x, y} H_{x, y}} \int|| F_{\text {retr }}|-| F_{\text {in }}\right|^{2} \mathrm{dr} / \sum_{E_{x, y} H_{x, y}} \int\left|F_{\text {in }}\right|^{2} \mathrm{dr}$, where $F$ indicates the electric and magnetic field components of the retrieved (retr.) and input (in) fields.

\section{SP coordinate transformations}

The orientation of the $s p$-basis vectors is constructed from the in-plane wavevector according to

$$
\begin{aligned}
& \hat{\mathbf{s}}=\hat{\mathbf{k}} \times \hat{\mathbf{z}} \\
& \hat{\mathbf{p}}_{ \pm}=\frac{\kappa \hat{\mathbf{z}} \mp k_{z} \hat{\mathbf{k}}}{k_{0}}
\end{aligned}
$$

where $k_{z}=\sqrt{k_{0}^{2}-\kappa^{2}}$. In our experiment, there are only upward-propagating fields, and we use the following equations to convert the fields in the $s p$-basis to those in a Cartesian basis,

$$
\begin{aligned}
& E_{x}(\mathbf{\kappa})=\sin \phi E_{s}(\mathbf{\kappa})-\frac{k_{z}}{k_{0}} \cos \phi E_{p}(\mathbf{\kappa}) \\
& E_{y}(\mathbf{\kappa})=-\cos \phi E_{s}(\mathbf{\kappa})-\frac{k_{z}}{k_{0}} \sin \phi E_{p}(\mathbf{\kappa}) \\
& H_{x}(\mathbf{\kappa})=\sin \phi \frac{E_{p}(\mathbf{\kappa})}{Z_{0}}+\frac{k_{z}}{k_{0}} \cos \phi \frac{E_{s}(\mathbf{\kappa})}{Z_{0}} \text { and } \\
& H_{y}(\mathbf{\kappa})=-\cos \phi \frac{E_{p}(\mathbf{\kappa})}{Z_{0}}+\frac{k_{z}}{k_{0}} \sin \phi \frac{E_{s}(\mathbf{\kappa})}{Z_{0}}
\end{aligned}
$$

These equations are derived in Supplementary Note 5 and can be straightforwardly inverted to find the transformation from a Cartesian to an $s p$-basis.

\section{Acknowledgements}

The authors thank Irina Kabakova and Anouk de Hoogh for their help with the experiments and fabrication. The authors acknowledge the support from the European Research Council (ERC Advanced Grant 340438-CONSTANS). This work is also part of the research program Rubicon with project number 68050-1513, which is partly financed by the Netherlands Organization for Scientific Research (NWO). Finally, part of this work is also funded by the Natural Sciences and Engineering Research Council of Canada.

\section{Author details}

'Optical Materials Engineering Laboratory, ETH Zürich, 8092 Zurich, Switzerland. ${ }^{2}$ Center for Nanophotonics, AMOLF, Science Park 104, 1098 XG Amsterdam, The Netherlands. ${ }^{3}$ Institute for Optical Sciences, University of Toronto, 60 St. George Street, Ontario M5S 1A7, Canada. IInstitute of Science and Technology Austria, Am Campus 1, 3400 Klosterneuburg, Austria. ${ }^{5}$ Kavli Institute of Nanoscience, Department of Quantum Nanoscience, Delft University of Technology, Lorentzweg 1, 2628 CJ Delft, The Netherlands. ${ }^{6}$ Niels Bohr Institute and Center for Hybrid Quantum Networks, University of Copenhagen, Blegdamsvej 17, DK-2100 Copenhagen, Denmark

\section{Author contributions}

N.R., L.K., and J.S. conceived the study. J.E.S., N.R., B.I.F., and L.K. developed the deconvolution algorithm. B.I.F performed the numerical analyses and simulations. N.R., B.I.F, M.W., and L.K. performed the experiments. J.E.S., N.R., B.I F., and L.K. wrote the manuscript.

\section{Competing interests}

The authors declare no competing financial interests.

Supplementary information is available for this paper at https://doi.org/ 10.1038/s41377-019-0124-3.

Received: 2 June 2018 Revised: 17 December 2018 Accepted: 7 January 2019

Published online: 06 March 2019

\section{References}

1. Sersic, I. et al. Electric and magnetic dipole coupling in near-infrared split-ring metamaterial arrays. Phys. Rev. Lett. 103, 213902 (2009).

2. Ryabov, A. and Baum, P. Electron microscopy of electromagnetic waveforms. Science. 353, 374-377 (2016).

3. Yen, T. J. et al. Terahertz magnetic response from artificial. Mater. Sci. $\mathbf{3 0 3}$ 1494-1496 (2004).

4. Staude, I. et al. Tailoring directional scattering through magnetic and electric resonances in subwavelength silicon nanodisks. ACS Nano 7, 7824-7832 (2013).

5. Zywietz, U. et al. Laser printing of silicon nanoparticles with resonant optical electric and magnetic responses. Nat. Commun. 5, 3402 (2014).

6. Geffrin, J. M. et al. Magnetic and electric coherence in forward- and backscattered electromagnetic waves by a single dielectric subwavelength sphere. Nat. Commun. 3, 1171 (2012).

7. Rotenberg, $\mathrm{N}$. et al. Tracking nanoscale electric and magnetic singularities through three-dimensional space. Optica 2, 540-546 (2015).

8. García de Abajo, F. J. Colloquium: light scattering by particle and hole arrays. Rev. Mod. Phys. 79, 1267-1290 (2007)

9. Tang, Y. \& Cohen, A. E. Optical chirality and its interaction with matter. Phys. Rev. Lett. 104, 163901 (2010)

10. Bliokh, K. Y. \& Nori, F. Characterizing optical chirality. Phys. Rev. A 83, 021803 (2011).

11. Bliokh, K. Y., Bekshaev, A. Y., and Nori, F. Extraordinary momentum and spin in evanescent waves. Nat. Commun. 5, 3300 (2014).

12. Bliokh, K. Y., Smirnova, D. \& Nori, F. Quantum spin Hall effect of light. Science 348, 1448-1451 (2015).

13. Bliokh, K. Y. et al. Spin-orbit interactions of light. Nat. Photonics 9, 796-808 (2015).

14. Betzig, E. \& Chichester, R. J. Single molecules observed by near-field scanning optical microscopy. Science 262, 1422-1425 (1993). 
15. Burresi, M. et al. Probing the magnetic field of light at optical frequencies. Science 326, 550-553 (2009).

16. Denkova, D. et al. Mapping magnetic near-field distributions of plasmonic nanoantennas. ACS Nano 7, 3168-3176 (2013).

17. Caselli, N. et al. Deep-subwavelength imaging of both electric and magnetic localized optical fields by plasmonic campanile nanoantenna. Sci. Rep. 5, 9606 (2015).

18. Vignolini, S. et al. Magnetic imaging in photonic crystal microcavities. Phys. Rev. Lett. 105, 123902 (2010).

19. le Feber, B. et al. Simultaneous measurement of nanoscale electric and magnetic optical fields. Nat. Photonics 8, 43-46 (2014).

20. Rotenberg, N. et al. Plasmon scattering from single subwavelength holes. Phys. Rev. Lett. 108, 127402 (2012).

21. Bauer, T. et al. Nanointerferometric amplitude and phase reconstruction of tightly focused vector beams. Nat. Photonics 8, 23-27 (2014).

22. Olmon, R. L. et al. Determination of electric-field, magnetic-field, and electriccurrent distributions of infrared optical antennas: a near-field optical vector network analyzer. Phys. Rev. Lett. 105, 167403 (2010).

23. Grosjean, T. et al. Full vectorial imaging of electromagnetic light at subwavelength scale. Opt. Express 18, 5809-5824 (2010).

24. Engelen, R. J. P. et al. Subwavelength structure of the evanescent field of an optical bloch wave. Phys. Rev. Lett. 102, 023902 (2009).

25. Veerman, J. A. et al. Single molecule mapping of the optical field distribution of probes for near-field microscopy. J. Microsc. 194, 477-482 (1999).
26. de Fornel, F. et al. Analysis of image formation with a photon scanning tunnelingmicroscope. J. Opt. Soc. Am. A 13, 35-45 (1996).

27. Greffet, J.-J. \& Carminati, R. Image formation in near-field optics. Prog. Surf. Sci. 56, 133-237 (1997).

28. Schmidt, S. et al. Image formation properties and inverse imaging problem in aperture based scanning near field optical microscopy. Opt. Express 24 4128-4142 (2016)

29. Esteban, R. et al. Direct near-field optical imaging of higher order plasmonic resonances. Nano Lett. 8, 3155-3159 (2008).

30. Kabakova, I. V. et al. Imaging of electric and magnetic fields near plasmonic nanowires. Sci. Rep. 6, 22665 (2016).

31. Burresi, $M$. et al. Magnetic light-matter interactions in a photonic crystal nanocavity. Phys. Rev. Lett. 105, 123901 (2010).

32. Lodahl, P. et al. Chiral quantum optics. Nature 541, 473 (2017).

33. Wang, M., et al. Magnetic spin-orbit interaction of light. Light Sci. Appl. 7, 24 (2018).

34. Schuller, J. A. et al. Plasmonics for extreme light concentration and manipulation. Nat. Mater. 9, 193-204 (2010).

35. Koenderink, A. F., Alù, A. \& Polman, A. Nanophotonics: shrinking light-based technology. Science 348, 516-521 (2015).

36. Kildishev, A. V., Boltasseva, A., and Shalaev, V. M. Planar Photonics with Metasurfaces. Science 339, 6125 (2013).

37. Aigouy, L. et al. Mapping and quantifying electric and magnetic dipole luminescence at the nanoscale. Phys. Rev. Lett. 113, 076101 (2014). 